Kansas State University Libraries

New Prairie Press

\title{
DISTANCE MEASURES IN POST HOC COMPARISONS OF TEMPERATURE GERMINATION QUADRATIC RESPONSE SURFACES
}

D. E. Palmquist

S. N. Bagchi

J. A. Young

R. D. Davis

See next page for additional authors

Follow this and additional works at: https://newprairiepress.org/agstatconference

Part of the Agriculture Commons, and the Applied Statistics Commons

\section{(c) (1) $\Theta($}

This work is licensed under a Creative Commons Attribution-Noncommercial-No Derivative Works 4.0 License.

\section{Recommended Citation}

Palmquist, D. E.; Bagchi, S. N.; Young, J. A.; and Davis, R. D. (1993). "DISTANCE MEASURES IN POST HOC COMPARISONS OF TEMPERATURE GERMINATION QUADRATIC RESPONSE SURFACES," Conference on Applied Statistics in Agriculture. https://doi.org/10.4148/2475-7772.1370

This is brought to you for free and open access by the Conferences at New Prairie Press. It has been accepted for inclusion in Conference on Applied Statistics in Agriculture by an authorized administrator of New Prairie Press. For more information, please contact cads@k-state.edu. 
Author Information

D. E. Palmquist, S. N. Bagchi, J. A. Young, and R. D. Davis 


\title{
DISTANCE MEASURES IN POST HOC COMPARISONS OF TEMPERATURE- GERMINATION QUADRATIC RESPONSE SURFACES
}

\author{
D.E. Palmquist ${ }^{1}$ S.N. Bagchi ${ }^{2}$ J.A. Young, and R.D. Davis \\ ${ }^{1}$ USDA/ARS, 920 Valley Rd., Reno, NV 89512, and \\ ${ }^{2}$ Dept. of Mathematics, University of Nevada Reno
}

\begin{abstract}
Generalized quadratic response surface models are used to describe seed germination at diurnally alternating cold and warm incubation temperatures for three Great Basin exotic plant species. The method of the F-statistic $\mathrm{SS}_{\mathrm{drop}}$ is applied to determine whether the response surface models are equal. Two proposed distance measures are used as modified multiple comparison techniques for determining differences between surfaces. These measures prove useful in distinguishing between the species showing the highest germination response and the one showing the lowest response to the incubation temperature ranges studied.
\end{abstract}

\section{Introduction and Literature Review}

Many phenomena in the biological sciences which show growth, stabilization, and reduction are best represented by equations which describe these characteristic growth patterns. Seed germination as a function of incubation temperature is such a phenomenon. The generalized quadratic response surface model describes the typical emergence of plant seeds subjected to alternating day and night incubation temperatures (Evans et al. 1982). There are ranges of temperatures where optimal emergence occurs. Above and below these optimal temperature ranges, emergence is inhibited. Different plant species have differing optimal incubation temperature ranges. A quadratic equation can be used to show this relationship of emergence inhibition at temperatures that are either too low or too high to support emergence while providing an approximation of the relation between emergence and temperature in the optimal range for a particular plant species.

While a quadratic form of equation is useful for describing the relationship between seedling emergence and incubation temperatures for a particular plant species, researchers are often interested in comparing the performance of one plant species to that of another. A question to consider in revegetation experiments after wildfires is which plant species are best suited to emerging and surviving in these altered conditions (Evans and Young 1984). Many mining companies are having to obey government mandates to return areas destroyed by their activities to a condition that is as close to their original condition as possible. The need to compare the response of one plant species to those of others in determining which ones are best suited for revegetation helps ensure the best chance of successful reestablishment.

Statisticians and scientists who apply statistical techniques have been concerned about the proper tests to use when comparisons between treatments with quantitative factor levels are of interest to the experimenter. The most common use of multiple comparison procedures is to make all pairwise comparisons of treatment means for qualitative factor levels such as plant varieties (or cultivars), locations, types of fertilizers used, etc. (Chew 1976). Their purpose is to detect possible groups or groupings among a set of unstructured treatments (Petersen 1977). These techniques are not only inappropriately applied to experiments where quantitative levels of factors are involved and regressions have been conducted, but the information supplied by standard multiple comparison techniques does not address the same questions that are being asked in the regression context. When comparing the emergence 
(or germination) response of one plant species to others, an experimenter may want to know if the different plant species respond in a similar fashion to temperature fluctuations in the environment, not whether the mean germination response from one plant species equals that of another over applied treatments. A reasonable question to ask is whether the functional responses of germination between species are similar for varying levels of day and night temperature ranges. Furthermore, if it can be shown that different plant species do have differing germination responses to incubation temperatures, another question is which plant species have similar responses and which do not. A general linear test approach for determining the equality of two or more response surfaces using the partial F-test already exists (Neter and Wasserman 1974). While there is extensive literature on comparing intercepts and slopes for simple linear regressions, the procedure becomes more difficult as the number of comparisons increases or when testing multiple regressions (Neter and Wasserman 1990). The same problems exist as those inherent in the use of multiple t-tests when the partial F-test technique is used to make all pairwise comparisons. After extensive literature reviews, no information was found that tackled the particular problem of multiple comparisons between regression equations. Assuming that the partial

F-test has been performed and the null hypothesis of no differences between the surfaces is rejected, we know that at least one surface is different from the rest. There is no underlying distribution which provides information on how far apart two surfaces have to be in order to be declared significantly different. We propose the use of two distance measures for comparing different plant species' emergence responses to incubation temperature when a multiple regression approach is taken.

It is proposed that the only statistically significant difference that can be obtained between the surfaces tested is the one between the pair of surfaces showing the largest values of the proposed indices. These indices can be used to distinguish two different concepts of distance between the plant species tested. One proposed measure (or index) gives information on how similar two plant species' emergence responses are for the incubation temperature ranges studied. This index assumes that the initial emergence for the two species is the same. The other index provides information that includes the initial and subsequent emergence response differences between the two plant species being compared.

\section{Methods of Data Analysis}

Generalized quadratic response surface models are used to describe emergence at alternating cold and warm incubation temperatures for three introduced Great Basin plant species. The general quadratic form of the response surfaces used in this analysis is:

$Z=\beta_{0}+\beta_{1} \star X+\beta_{2} \star Y+\beta_{3} \star X^{2}+\beta_{4} \star Y^{2}+\beta_{5} \star X \star Y$

where :

$\begin{array}{lll}Z & = & \text { estimated percent emergence, } \\ \beta_{0} & = & \text { intercept, } \\ \beta_{1}-\beta_{5} & = & \text { partial regression coefficients for the } \\ \text { variables } \mathrm{X} \text { and } \mathrm{Y}, & \\ \mathrm{X} & =\quad \text { cold (night) temperature varied between } 0 \text { and } \\ \mathrm{Y} & =\text { warm (day) temperature varied between } \mathrm{X} \text { and } 40^{\circ} \mathrm{C} .\end{array}$

Separate $2^{\text {nd }}$ order models are fit to each of the 3 plant species. This is followed by fitting another $2^{\text {nd }}$ order model combining the data for all 3 plant species. A partial F-test, hereinafter referred to as the $\mathrm{SS}_{\mathrm{drop}}$ statistic or $S_{S_{\text {drop }}}$ test, is then performed. If it is determined that there are differences between the 3 plant species response surfaces, we proceed 
to calculate our distance measures (indices), which are then used in a modified multiple comparison procedure.

\section{Distance Using Cosine}

Rewriting the general quadratic response surface model into the form of a hyperplane in $\mathbf{R}^{6}$ yields the general equation:

$$
\beta_{1} \star X+\beta_{2} \star Y+\beta_{3} \star X^{2}+\beta_{4} \star Y^{2}+\beta_{5} \star X * Y-Z=-\beta_{0}
$$

The $\beta$ coefficient vector for the general equation (written in transpose) is: $\left[\beta_{1}, \beta_{2}, \beta_{3}, \beta_{4}, \beta_{5},-1\right]$. The first index that we propose is a measure of the angle between the coefficient vectors in $\mathbf{R}^{6}$ for the two response surfaces. It is defined as:

$$
A=1-|\cos \theta|, \quad 0 \leq A \leq 1
$$

where $\cos \theta=\left.\frac{\left(\beta^{(i)} \beta^{(j)}\right)}{\left|\beta^{(i)}\right| \beta^{(j)}}\right|^{\prime} \quad 0 \leq \theta \leq \pi$,

and $\beta^{(n)}$ is the vector of coefficients that defines a hyperplane in $\mathbf{R}^{6}$ for plant species $n$ from its corresponding quadratic response surface. $\left(\beta^{(i)}, \beta^{(j)}\right)$ is the standard inner product of the coefficient vectors $\beta^{(i)}$ and $\beta^{(j)}$, and $\beta^{(i)}$ is $\left(\beta^{(i)}, \beta^{(j)}\right)$. This measure treats each term in the quadratic regression model, except for the intercept, as a distinct variable. Hence, it transposes a three dimensional polynomial regression equation into a hyperplane in $\mathbf{R}^{6}$.

This index uses the standard mathematical concept of orthogonality to provide a measure of the orientation between two surfaces being tested. This is analogous to measuring the similarity of the plant species' rates of emergence to the temperature variables, without taking into account the effect of their intercepts. Two species may have the same rates of emergence response to temperature but be displaced from one another by a fixed amount of germination over the entire temperature range. By not using the information from the intercepts, this measure does not take into account this possible emergence/germination displacement between the plants.

\section{Distance Using $\mathrm{I}_{2}-$ Norm}

The second index we propose does measure the amount of displacement in germination between two plant species over the entire temperature range of testing. Let $f^{(i)}, f^{(j)}$ be the $i^{\text {th }}$ and $j^{\text {th }}$ quadratic response surface, respectively. This index is $D\left(f^{(i)}, f^{(j)}\right)$, where $D$ is the $L_{2}$ norm of $f^{(i)}-f^{(j)}$. It is defined here as:

$$
D\left(f^{(i)}, f^{(j)}\right)=\left[\int_{0}^{40} \int_{x}^{40} \frac{\left[f^{(i)}-f^{(j)}\right]^{2}}{800} d y d x\right]^{1 / 2}
$$

The integral is calculated over the range of cold and warm temperatures used in the study and is standardized over these temperature ranges. The index $D$ measures departure, specifically the shifting between two surfaces on the axis of the dependent variable, seedling emergence. Unlike the index $A$, the measure $D$ yields no information as to the similarity in overall emergence rates between the response surfaces of two plant species. 


\section{Advantages of Distance Measures}

Use of these indices involves no additional hypothesis testing, other than the $\mathrm{SS}_{\mathrm{drop}}$ statistic. If the $\mathrm{sS}_{\text {drop }}$ statistic is not significant, one concludes that there are no statistical differences between any of the response surfaces tested. If a significant SS $_{\text {drop }}$ statistic is obtained, one concludes that at least one of the surfaces is different from the rest. The proposed indices for each pair of surfaces are calculated, then ranked from highest to lowest, so that it is possible to obtain two different rankings (one for each index). We then take the most conservative approach by declaring the only significant difference to be between the pair of surfaces with the largest value of the proposed index.

The use of these measures as modified multiple comparison tests allows a researcher to avoid some problems associated with standard ANovA multiple comparison testing procedures. The probability of increased Type I errors as the number of surfaces being compared is increased is no longer a problem since no statistical significance is being associated with any of the surface pairs except for the two extremes (as measured by the

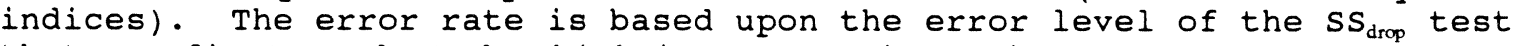
that was first conducted, which is an experimentwise error rate. The rest of the surfaces can be visualized in relation to the two extremes, but no statistical significance can be attributed to their relationship with the extremes. The plant species pair with the largest value of the index $A$ is determined to have significantly different emergence response rates to incubation temperature. Similarly, the plant species pair with the largest $D$ index value is determined to have a significantly different amount of overall germination.

Three exotic Great Basin herbaceous plant species that dominate the secondary successional flora of the big sagebrush (Artemisia tridentata Nutt.) ecosystem were chosen for the study. Barbwire Russian thistle (Salsola paulsenii Lit.), alternately referred to as thistle, is an introduced weed found on the margins of salt deserts (Young and Evans 1979). Tumble mustard (Sisymbrium altissimum L.), alternately referred to as mustard, is a broadleaf weed that replaces Russian thistle in a seral continuum. Common mullein (Verbascum thapsus L.), alternately referred to as mullein, is a rural weed in the big sagebrush zone common to roadsides and industrial areas (Semenza et al. 1978). These three plant species were chosen because two of them, thistle and mustard, have similar emergence responses to temperature (Palmquist et al. 1987), while the third, mullein, has differing emergence responses from the other two (Evans et al. 1982). We wanted to test the proposed distance measures to see if they could demonstrate the same relationships among the three species that we knew existed before the experiment.

\section{Results and Discussion}

\section{Individual Response surfaces}

The quadratic response surface equations for estimated percent emergence, along with their corresponding $R^{\frac{1}{2}}$ values, for the three species are:

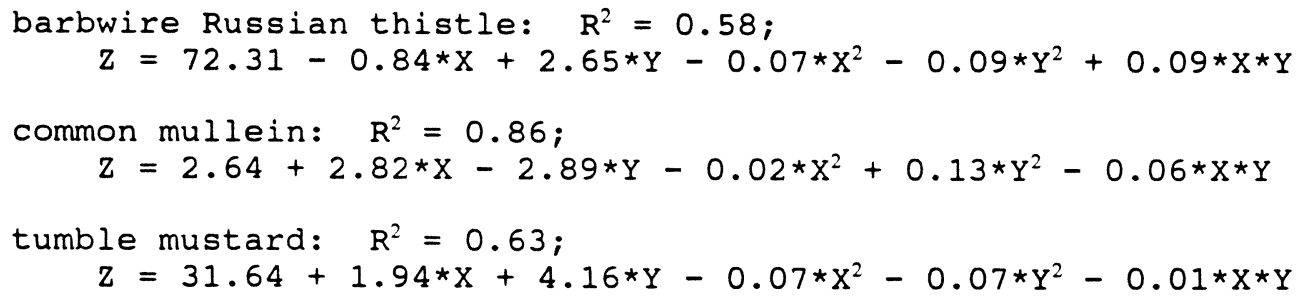


Restraints on the above equations were fitted for emergence at 0 to $100 \%$. Emergence, or germination, is classified by the use of distinct seedbed temperature categories based on ongoing microenvironmental monitoring research in the field (Evans et al. 1970; Evans and Young 1970; Evans and Young 1972). Using this classification system (fig. 1a), a plant species can be characterized as being either a cold temperature germinator, a moderate temperature germinator, a warm temperature germinator, or a fluctuating temperature germinator, based on which specific seedbed temperature area supports optimum emergence. Barbwire Russian thistle is classified as a high germinator at cold and moderate temperatures with good overall emergence in the other temperature ranges ( $\mathrm{fig}$. $1 \mathrm{~b}$ ). Common mullein is an extremely high percentage germinator at the warmest end of the fluctuating and warm temperature ranges and shows very low germination at the colder temperatures (fig. 1c). Tumble mustard is a high percentage germinator in the moderate and fluctuating temperature categories, falling off somewhat in the colder temperature ranges (fig. 1d).

\section{Response Surface Comparisons}

The general linear test for the equality of the three response surfaces yields the result that there are indeed differences between them ( $F^{*}$ $233.6, p \leq 0.01)$. Proceeding to calculate the values of each index for all pairwise combinations of the surfaces produces the results found in Table 1. It happens that the response surface pair of mustard and mullein has the largest value for both indices. As measured by index $A$, we conclude that these two plant species' emergence responses to the incubation temperature variables are significantly different from one another $(p \leq 0.01)$. They also have the largest displacement between any two plant species' response of overall emergence, as measured by index $D$.

Even though no statements of significance can be attributed to the plant species whose index values are not the largest, it is possible to visualize the relationships between these species and the ones declared significantly different. It appears that the overall displacement of the emergence responses of thistle and mustard is not very large over the temperature ranges studied (index $D$ Table 1). The displacement in emergence responses between thistle and mullein is almost as high as that between mustard and mullein (Table 1). According to index $D$, the displacement between the response surfaces in $\mathbf{R}^{3}$, thistle and mustard are the most similar in their overall amounts of germination over all the incubation temperatures used in the study. Mustard and mullein have the largest germination differences over the incubation temperatures, and hence are significantly different from one another. Thistle and mullein also have large differences in the amount of germination occurring over the incubation temperatures, which are probably not significantly different from that of mustard and mullein, although no significance statement can be attributed to this difference.

On examining the actual angle between the hyperplanes for these species in $\mathbf{R}^{6}$, we find the angle between thistle's hyperplane and mullein's hyperplane to be smaller than that between thistle and mustard (Table 1). But, we also find that thistle and mustard have positively oriented hyperplanes in $\mathbf{R}^{6}$, while thistle and mullein have negatively oriented hyperplanes in $\mathbf{R}^{6}$. Index $A$ and information about the positive or negative orientation of the hyperplanes in $\mathbf{R}^{6}$ show that thistle and mustard have the closest positive relationship. Mustard and mullein are the most dissimilar, hence, have significantly different emergence response rates to incubation temperature.

\section{Summary and Conclusions}

The partial F-statistic, $\mathbf{S S}_{\text {drop }}$ method, is the standard method used in determining whether or not differences exist between two or more multiple 
regression equations with the same functional form. When testing more than two equations, the results of a significant F-ss drop test tell that at least one of the equations tested is different from the others. We propose using two distance measures as a modified multiple comparison technique that would show which pair of multiple regression equations in the study are significantly different when a significant F-statistic is obtained. The two measures are:

1) D: the overall shift in the $\mathrm{z}$-axis response between two regression equations, and

2) A: a measure based on the angle between the hyperplanes of the two regression equations in $\mathbf{R}^{6}$.

The $L_{2}$ norm metric, $D$, provides information about the significance of the displacement between two equations, while the angle measure, $A$, provides information on the similarity of the overall rate of response between two equations. Because we recommend that the only significantly different regression equations be defined as the pair with the largest values of the proposed indices, we avoid some of the problems associated with the standard multiple comparison techniques used in ANOVA designs.

We used the proposed distance measures as a modified multiple comparison technique to compare between quadratic response surfaces. This enabled us to substantiate previously known relationships of seedling emergence to incubation temperature between the plant species we tested. We were able to further explore some of the physical differences between the shapes of the surfaces, such as displacement from one another and overall response rate similarity.

The methodology for use of the two proposed distance measures is not limited to a quadratic form in two variables. It is valia for comparing between any two or more multiple regression equations with the same general linear model form.

Future work remains to be done in identifying a distribution that would allow us to perform all pairwise response surface comparisons, instead of identifying just the two extreme surfaces. 


\section{Applied Statistics in Agriculture}

WARM PERIOD $8 \mathrm{hr}{ }^{\circ} \mathrm{C}$

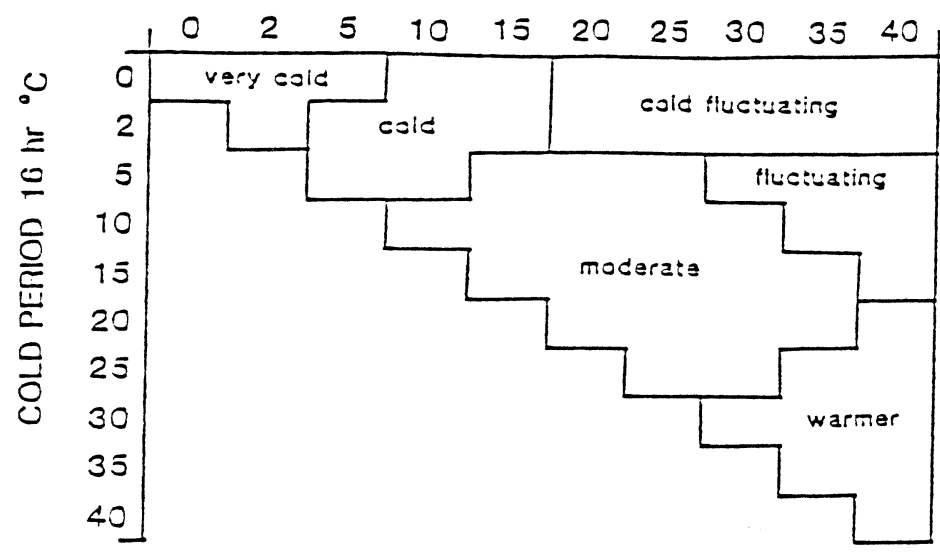

Fig. la. General temperature categories of seed germination based on monitoring studies.

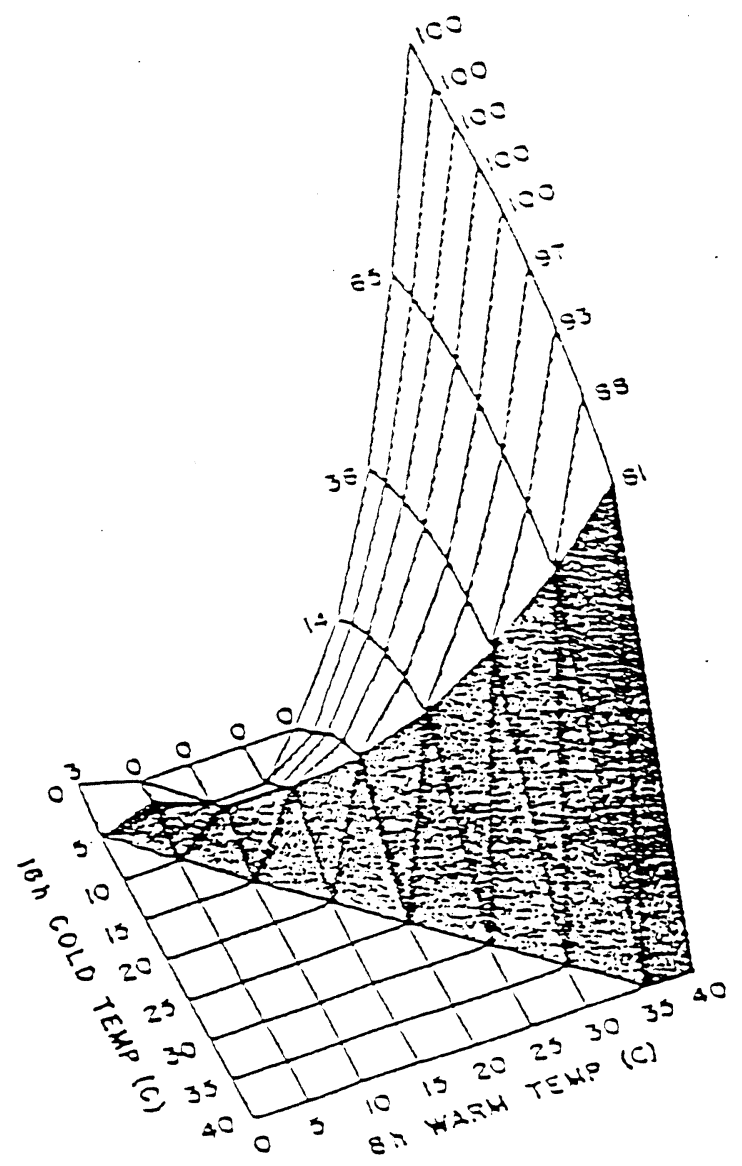

Fig. 1c: Quadratic response surface of common mullein.

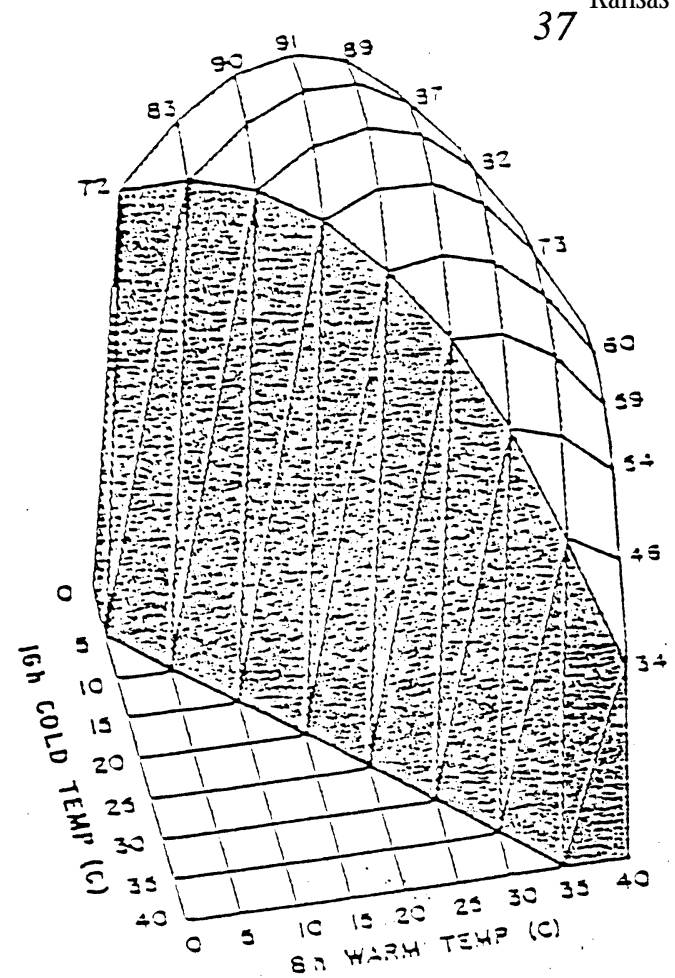

Fig. 1b. Quadratic response surface of Barbwire Russian thistle emergence,

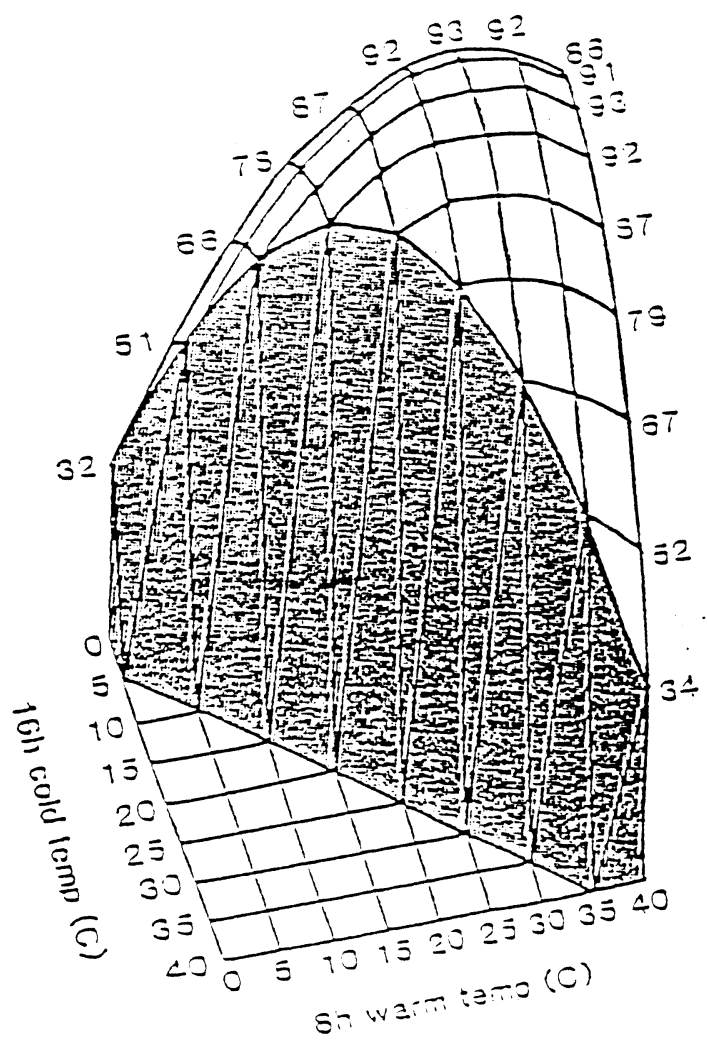

垈)

Fig. 1d. Quadratic response surface of tumble mustard. 

Table 1. Values of the indices $A$ and $D$, and the angle of their coefficient vector hyperplanes in $\mathbf{R}^{6}$, calculated for all pairwise combinations of response surfaces for the different plant species.

$\begin{array}{lccc}\text { Plant Species Pair } & \text { Index A } & \text { Index D } & \text { Anqle (deg) } \\ \text { Thistle - Mustard } & 0.264 & 19.46 & 42.6 \\ \text { Thistle - Mullein } & 0.107 & 56.62 & 153.2 \\ \text { Mustard - Mullein } & 0.647^{*} & 58.93^{*} & 110.7\end{array}$

* Largest index value of all the comparisons indicating significant difference $(p \leq 0.01)$ between the response surfaces of the corresponding plant species. 


\section{Literature cited}

Chew, V. 1976. Comparing treatment means: A compendium. Horticultural Science 11:348-357.

Evans, R.A., R. Holbo, R.E. Eckert, Jr., and J.A. Young. 1970. Functional environment of downy brome communities in relation to weed control and revegetation. Weed science 18:154-162.

Evans, R.A., and J.A. Young. 1970. Plant litter and establishment of alien annual weed species in rangeland communities. Weed science $18: 697-703$.

Evans, R.A., and J.A. Young. 1972. Microsite requirements for establishment of annual rangeland weeds. Weed science 20:350-356.

Evans, R.A., D.A. Easi, D.N. Book, and J.A. Young. 1982. Quadratic response surface analysis of seed-germination trials. Weed Science $30: 411-416$.

Evans, R.A., and J.A. Young. 1984. Microsite requirements for downy brome (Bromus tectorum) infestation and control on sagebrush rangelands. Weed Science 32 (Supp. 1):13-17.

Neter, J. and W. Wasserman. Applied Linear Statistical Models. 1974. Richard D. Irwin, Inc. Homewood, IL. 842 p.

Neter, J. and W. Wasserman. Applied Linear Statistical Models. $3^{\text {rd }}$ Edition. 1990. Richard D. Irwin, Inc. Homewood, IL. 1181 p.

Palmquist, D.E., R.A. Evans, and J.A. Young. 1987. Comparative analysis of temperature-germination response surfaces. Proceedings of Symposium "Seed and seedbed Ecology of Rangeland Plants", Tucson, AZ. pp. 97-103.

Petersen, R.G. 1977. Use and misuse of multiple comparison procedures. Agronomy Journal 69:205-208.

Semenza, R.J., J.A. Young, and R.A. Evans. 1978. Influence of light and temperature on the germination and seedbed ecology of common mullein (Verbascum thapsus). Weed science 26:577-581.

Young, J.A., and R.A. Evans. 1979. Barbwire Russian thistle seed germination. Journal of Range Management 32:390-394. 